\title{
Evidence Based Decision Making: A Review
}

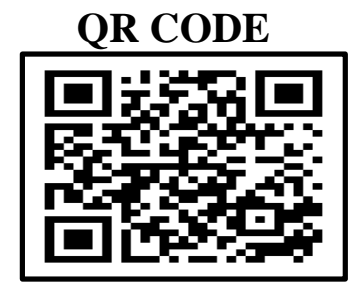

\section{TWESHA HUIDROM*1, RAVNEET MALHI ${ }^{20}$}

In today's era, many of the decisions are based upon gut feeling, intuition, or instinct rather than relied on actual scientific data and facts. It's important and essential to do the needful based on facts and not feelings for the best possible outcomes. Evidence based decision making in the field of healthcare describes the integration of basic rules of evidence as they evolve into implementation in daily practices. In dentistry, the principles of evidence-based decision making as well as a very common clinical dilemma to make a decision on whether to save and preserve the natural tooth or to extract and replace it with an implant or any sort of prosthesis is often experienced by a clinician easily. The need of evidence plays a very important role in making decisions. It helps provide a better outcome which will result in fewer casualties.

KEYWORDS: Evidence-Based, Medicine, Dentistry, Decision, Error

\section{INTRODUCTION}

"Human history is mostly the story of error and accident."

-Michael A. Ledeen

In today's advanced era, many of the decisions are based upon gut feeling, intuition, or instinct rather than relied on actual scientific data and facts. Though presence of facts and figures is already available but due to the lack of an individual's interest and resistance towards information it results in errors and problems. Everyone, be it a person, a society or an establishment has made errors in the form of imprecise beliefs or decisions. In earlier times, errors and mistakes in evidence-based decision making must have been greater than that in the recent times. Before, the research and the findings were far lesser than that which we can find today. ${ }^{1}$ Written material is widely accepted and is like the Holy Grail for evidence-based decision making. It gets updated and new research findings are added so that errors can be decreased giving us better results. Errors can occur anywhere in the process of decision making. It can be on an individual level by considering beliefs and interpretation of information on their own and can result in inaccurate application of the process. Disposition and aversion to information can also result in errors while making decisions. Our main aim is to identify and reduce these errors so that the quality and quantity of the result is not hampered.
The categories which can be included for improvement are basically reduction in time, cost, effort, dispute, disease and many other possibilities. ${ }^{2}$ We know that eliminating all the errors in one go is not possible. But the severity and the frequency can be reduced and should be considered our main goal. Some of the prerequisites which should be followed at an individual level include accepting the fact that there may be errors in any evidence; the individual should be willing to identify the errors and lastly they have to accept that research attempts to obtain justifiable facts and evidence. ${ }^{3}$

Thus, it's important and essential to do the needful based on facts and not feelings for the best possible outcomes. Evidence-based decision making (EBDM) is an ideal model which we can use to ensure that you are considering relevant facts and not something that is not proven. Evidence based decision making is a process that helps in making decisions about a program, practice, or policy that is grounded in the best available research evidence and informed by experiential evidence from the field and relevant contextual evidence. All the information present in scientific research with good outcome should always considered while taking a decision. Three categories which are mainly the part of this framework are contextual evidence, best available research evidence and experiential evidence. ${ }^{4}$ 
The decision-making process has three main stages. First is the collection of evidence which basically means gathering evidence from the best available research. Second is the interpretation of the evidence. Third and the final stage are applying the things that we have learned from the evidence. In this we are considering all three stages while making the decision based on the evidence to prevent any issue or problem before it occurs. The characteristics of the decision-making process includes transparency, participation, openness, skilled leadership, facilitation and a definite process. We need to get the best outcomes using these characteristics. We need to know for a fact that every scenario is unique and the evidence differs from situation to situation. ${ }^{5}$

Evidence Based Decision Making in Medical Field: Historically, evidence based medicine goes back to the 17oos, though it was not clearly explained and advanced until the early 1980s. Evidence based medicine was initially known as 'critical appraisal' to describe the integration of basic rules of evidence as they evolve into implementation in daily practices. Evidence based medicine is defined as a conscientious, explicit and judicious use of current best evidence in making decisions about the care of individual patients. ${ }^{6}$ They are defined based on 4 important and basic categories, which involve acknowledgment of the patient's problem and making of a structured and well layered clinical question, thorough research in medical literature to bring out the best available evidence to answer the question, critical appraisal of all available evidence and usage of the evidence with all facets and contexts of the clinical conditions. Recent evidence-based decision making research has indicated that the framework and functioning of healthcare facilities has contributed to an organization's functional success by improving and increasing safety, standard and their regulation process. There is very little information accessible about the financial returns of evidence-based investments; such investments are eliminated during the decision-making process. ${ }^{6,7}$

Healthcare facilities are very costly, dangerous, inefficient and not easily accessible in many places. Though the government has provided many provisions for the betterment of healthcare in places where easy access of healthcare is hard but awareness in a majority of population is still lacking. Many issues arise from a good amount of people ignoring all the pleas from the designated healthcare workers. ${ }^{5}$ The evidence that has to be collected from the population is decreased due to this issue. Without surveying and collecting evidence, research in that field is going to be a very difficult process. Surveys and research are a very important source of information and updates which are accessed and used by the healthcare professionals to help them in decision making. Updating of such research and content plays a very major role in decision making. In a field where past research with proof and evidence tends to the need to have good results and also good prognosis of the individual, evidence-based decision making is really necessary. Majority of the healthcare professionals are survived by such written material. If the content is written and widely accepted with proof of it being mostly successful, then it is easily considered in the process of implementing the evidence into the treatment or decision making. In a health care setting, be it a private clinic or a hospital, the complicated cases which have a multifaceted treatment process, require an extensive list of evidences and maybe more professionals from a different medical field to create a treatment plan which will give the best prognosis and even help save a life. ${ }^{4,6,7}$

Sometimes it is possible that going by the book and following the protocols may turn out to be a risk for the patient and he or she may even lose his or her life. So, we do know for a fact that evidence in a lot of cases may not turn out to be useful or helpful for the patient. It can be the worst decision of his or her life and also the medical practitioner who is the one taking the decision will be at a tight spot from that point onwards. There are a lot of things that the medical practitioner has to explain and comply with after taking a decision based on the facts and figures, which in turn didn't help in preserving one's life. Evidence based decision making also helps in medico-legal issues. ${ }^{8}$ In very rare cases the gut feeling of a medical healthcare professional works wonders with the help of evidence. Like if the doctor has all the necessary reports and he knows his way to go about for the treatment but still chooses a different way based on his feeling and intuition, it can turn out be a really bad decision for the patient's life. There's a 5050 chance when feelings and intuition are followed. But there is $80-90 \%$ of a chance that the treatment goes well if we go by the evidence and follows all the protocols. Medical field is literally so vast and big. The need of evidences, research and updating of the research is a sole necessity to make decisions in the 
medical field. ${ }^{4,6,8,9}$

Evidence Based Decision Making in Dentistry: Usually in dentistry the complicated cases of endodontology, periodontology and oral rehabilitation often pose a dilemma in the clinician's mind as to how they can tackle the situation to give the correct treatment plan. The principles of evidence-based decision making as well as a very common clinical dilemma to make a decision on whether to save and preserve the natural tooth or to extract and replace it with an implant or any sort of prosthesis such as a fixed partial denture is often experienced by a clinician easily. Evidence through investigations and research can prove to provide better closure for the treatment plan. ${ }^{9}$ This will ultimately help in the better diagnosis of the treatment that has to be rendered to the patient. Many cases require a multifaceted treatment approach for the prognosis to be good with least number of complications. The outcome of the treatment may not be compromised if the main goals of the treatment are achieved. However, in a lot of cases where complications hamper the main treatment goals, decrease in predictable outcome is seen or anticipated. The rule of the thumb or the main principle in dentistry is to put a good amount of effort in preserving the natural teeth. Any different treatment outcome may lead to confusion when we try and compare two different treatment alternatives.

Therefore, it should be fully based on the correct measurements of the different treatments. Other factors like patient's preference, prosthetic/periodontal/endodontic considerations or long-term prognosis should be considered and should be recognized in the clinician or practitioner's final decision making. ${ }^{10,11}$

In a dental practice setting the use of clinical practice guidelines and protocols organized by the decision trees is the most ethical and successful way to go about in that setting? Now what is a decision tree is the question? A decision tree assists the dentist when he or she is presenting with the conditions and patient values are less possible in recommending treatment manners whose benefits and harms are varying and unknown. Best proof based on the 'average patient' is incorporated in the decision tree. Chances, utility and economic data are calculated and surveyed in providing the patient with informed and personalized choices of care. Evidence-based decision-making accounts in a process in which an individual patient is enlightened of the best probable evidence and all pertinent options. The result of the process is that patient serviceability and cost apprehensions are fully addressed in enhancing the clinical decision. In dentistry, evidence-based decision making is a discipline that provides optimum, clear-cut evidence to dentists and their patients in shared decision making. ${ }^{9,12}$

In the recent scenario, dentists are being provided with the training of being able to adopt the role of translational researchers in developing evidence based dental practices. Realistically, evidence-based dentistry is not feasible in its current form for the purveying of labor intensive provisions that distinguish recent dental practice. Evidence based decision making also conceptualizes a team proposition in explaining issues and solutions to change the recent dental practice. All these changes integrate a practice that involves an electronic tabulation, centralized and generalized database, knowledge management software and manpower in optimizing effective and useful oral healthcare to dental patients. Scientific, pertinent evidence is important in clinical care, policy making, controversy resolution and law. As a result, evidence-based practice brings together relevant, trustworthy information by in order collecting, inspecting and transferring research findings into clinical, management and strategy areas. One of the main important things in evidence-based decision making is to assess the available evidence to judge what is bad and what is good or better. The available evidence should be graded by the strength of the proof and evidence. The levels of evidence are often considered when applying it. The hierarchy is usually followed because older information keeps getting updated and the newest evidence is often the best evidence. In these levels of evidence randomized trial controls are mostly considered as high level of evidence. In 'Oxford centre of evidence-based medicine' the hierarchical system of classifying scientific evidence is elaborated very well. It provides an evident comprehension regarding the scientific evidence. This may lead to surprising conclusions that may dispute common concepts and even show a reverse pyramid of scientific evidence. ${ }^{10,12,13}$

Conclusion: The need of evidence plays a very important role in making decisions. It helps provide a better outcome which will result in fewer casualties. We know that newer technologies and recent advancements in scientific researches' will add to the 
legitimacy of the evidence which will ultimately result in making better decisions. Better decisions may not always lead to an accurate outcome but in most cases it does help in achieving the goal which is required. We should work on reinforcing and confirming the already present research and findings so that referring the research by the book will result in very fewer casualties.

"The delivery of medical care is to do as nothing as possible"

House of God-1979

\section{REFERENCES}

1. Martin GP, McKee L, Dixon-Woods M. Beyond metrics? Utilizing 'soft intelligence' for healthcare quality and safety. Soc Sci Med. 2015;142:19-26. https://doi.org/10.1016/j.socscimed.2015.07.027

2. Mackey A., Bassendowski S. The history of evidence-based practice in nursing education and practice. J Prof Nurs. 2017;33(10):51-5. https://doi.org/10.1016/j.profnurs.2016.05.009

3. CDC. Injury Prevention \& Control: Division of Violence Prevention (Online Article). Available from: https://vetoviolence.cdc.gov. [Last assessed on $12^{\text {th }}$ July, 2021].

4. Gabbay J, Le May A. Practice-based evidence for healthcare: clinical mindlines. Abingdon: Routledge; 2011

5. Barends E, Rousseau D, Briner R. Evidence-based management: the basic principles. In: Kovner A, D'Aunno T, editors. Evidence-Based Management in
Healthcare: Principles, Cases and Perspectives. Health Administration Press; Chicago, IL: 2017. pp. 320

6. Masic I, MiokovicM ,Muhamedagic B. Evidence Based Medicine - New Approaches and Challenges. Acta Inform Med. 2008; 16(4): 219-25.

7. Eddy DM. Evidence-based medicine: a unified approach. Health affairs (Project Hope) 2005;24(1):917

8. Warren JI, McLaughlin M, Bardsley J. The strengths and challenges of implementing EBP in healthcare systems. Worldviews Evid Based Nurs. 2016;13(1):15https://doi.org/24. 10.1111/wvn.12149

9. Rackett DL, Richardson WS, Rosenberg W, Haynes RB. Evidence-based medicine: how to practice and teach. 2. ed. Edinburgh: Churchill-Livingstone, 2000 10. Tsesis I, Nemkowsky CE, Tamse E, Rosen E. Preserving the natural tooth versus extraction and implant placement: making a rational clinical decision. Refuat Hapeh Vehashinayim (1993) 2010;27(1):37-46:75.

11. Iqbal MK, Kim S. A review of factors influencing treatment planning decisions of single-tooth implants versus preserving natural teeth with nonsurgical end- odontic therapy. J Endod. 2008;34(5):519-29.

https://doi.org/10.1016/j.joen.2008.01.002

12. Hofer TP, Kerr EA, Hayward RA. What is an error? Eff Clin Pract. 2000;3(6):261-9.

13. Angelos P. Complications, errors, and surgical ethics. World J Surg. 2009;33(4):609-11. https://doi.org/10.1007/soo268-0o8-9914-o. 